\title{
Mathematical model of thermal processes in a biogas plant
}

\author{
Amur Fiapshev ${ }^{1, *}$, Olesya Kilchukova ${ }^{1}$, Yuriy Shekikhachev ${ }^{1}$, Marat Khamokov ${ }^{1}$, and \\ Luan Khazhmetov ${ }^{l}$ \\ ${ }^{1}$ Kabardino-Balkarian State Agrarian University, 360030, Tolstoy str., 185, Nalchik, Russia
}

\begin{abstract}
One of the promising areas of processing poultry and livestock waste is anaerobic digestion, which helps to prevent pollution of the natural environment, as well as to receive processing products such as gaseous fuel, biogas and highly effective biofertilizer. The use of plants for the production of biological gas as alternative sources of energy is largely determined by its design characteristics and the worked out technological regimes. The study was conducted with the aim of obtaining data on the effect of the main parameters of the biogas plant and the heat exchanger-agitator on the quality of its operation. This paper considers the thermal processes taking place in the biogas plant in which the mixing device and the heating element are combined into one unit, which allows heating and maintaining the given temperature regime more evenly due to the rotation of the heat exchanger and the transfer of biomass heat throughout the whole volume of the methane. As a result of theoretical studies of the processes of heat exchange and heat transfer taking place in the biogas plant, a mathematical model has been obtained that allows determining the distribution of the temperature of the biomass throughout the entire volume of the methane. It is established that the theoretical temperature homogeneity of the stirred medium is achieved by combining the heat exchanger and the mixing device into one unit, the design and technological parameters of which characterize the intensity of the forced motion of fermentable manure, while changing the value of thermal conductivity.
\end{abstract}

\section{Introduction}

One of the most acute problems of the development of both the farming industry and the entire agro-industrial complex is the problem of its effective energy supply. If traditional sources of energy are constantly paid much attention, many are rather skeptical about the use of non-traditional energy sources to the present day. In general, such an attitude was laid down both by the low cost of traditional energy resources and by the rather low scientific justification for improving the efficiency of non-traditional and alternative energy sources [1]. At present, the picture is changing towards increased attention to the use of alternative energy sources, which is more connected both with the increase in their efficiency and the

\footnotetext{
*Corresponding author: energo.kbr@rambler.ru
} 
increase in tariffs for traditional energy resources [2]. In this regard, the relevance of research on improving the efficiency of the use of non-traditional energy sources for farms has increased significantly. The use of such unconventional sources as the energy received from biotechnology can be considered among the most acceptable directions [3].

One of the promising areas for the processing of poultry and livestock waste is anaerobic digestion, which helps to prevent pollution of the environment, and also to receive processed products such as gaseous fuels, biogas and highly effective biofertilizers [4].

\section{Problem Statement}

In the laboratory "Alternative Energy" of the Kabardino-Balkar State Agrarian University, research works are carried out to design and optimize the operation modes of the biogas plant for agricultural enterprises [5].

The work is carried out in accordance with the thematic plan-task for carrying out scientific research work of the Kabardino-Balkar State Agrarian University on the order of the Ministry of Agriculture of Russia at the expense of the federal budget for 2015 (agreement No. 2769/13 of December 29, 2014) on the topic "Development of methodological recommendations for the design of systems for the removal, treatment, disinfection, storage, and disposal of manure and litter in the part of modern methods of processing and utilization of manure and litter" [6].

To intensify the process of fermentation and optimize the design and energy parameters of the methane tank, we suggest combining the agitator (stirrer) and the heating element into one unit, that is, the stirring device is simultaneously a heating element [7]. This combination allows heating and maintaining the given temperature regime more evenly due to the rotation of the heat exchanger and the transfer of heat to the biomass (substrate) throughout the whole onsite sewage facility, since the homogeneity of the temperature in the moving medium is directly related to the phenomena occurring in the thermal boundary layer, in contrast to all existing heat exchangers (water jacket, tubular fixed) that allow heating only limited areas, which leads to uneven heating [8].

Due to such a constructive design of the bioreactor, the metal content of the structure and the energy costs for maintaining the temperature regime are reduced. On the contrary, the biogas output volume is increased due to uniform heat exchange and biomass mixing throughout the bioreactor volume.

The design of the bioreactor includes a heat exchanger-mixer being made as a vertical tubular shaft with four blades made of chrome-molybdenum steel tubes. In this case, the blades having a bracketed shape are arranged symmetrically and rigidly attached to the vertical tubular shaft with the possibility of rotation in the horizontal plane [9].

The bioreactor shown in Figure 1 consists of a sealed heat-insulated housing (1) with a cover (2), branch pipes (3) and a biomass outlet (4), a biogas outlet (5), and a heat exchanger (6). Figure 2 shows the arrangement of the blades of the heat exchanger-mixer. The geometric parameters of the bioreactor are shown in Figures 3-5. 


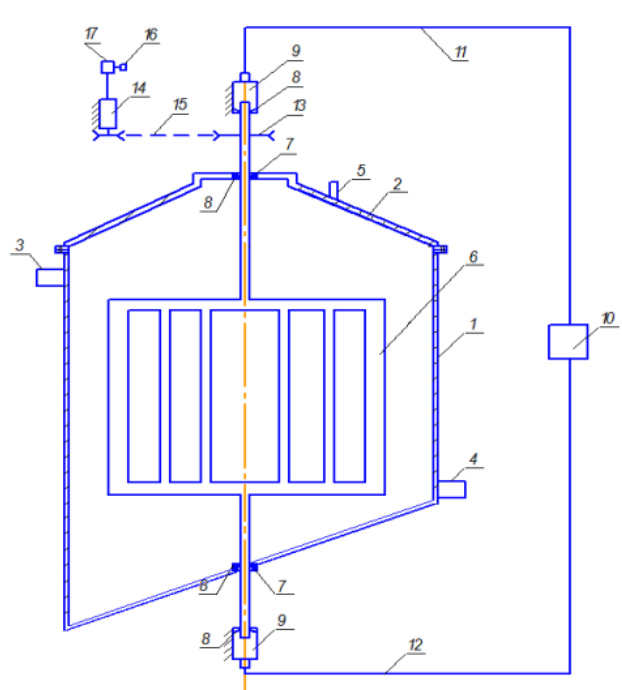

Fig. 1.

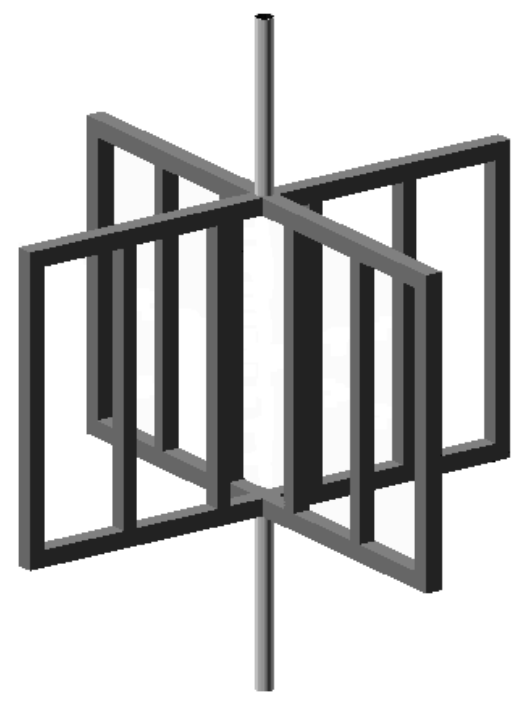

Fig. 2.

Fig. 1. The scheme of the bioreactor with heat exchanger-stirrer: 1 - body, 2 - cover, 3, 4 - branch pipes for supply and removal of biomass, 5 - branch pipes for biogas removal, 6 - heat exchanger for agitation, 7 - rolling bearing, 8 - stuffing box, 9 - cylindrical pipe, 10 - source of heat, 11,12 feed and return pipes, 13 - driven pulley, 14 - electric motor, 15 - V-belt drive, 16 - microprocessor controller, 17 - time relay software.

Fig. 2. The schematic layout of the blades of the heat exchanger-mixer.

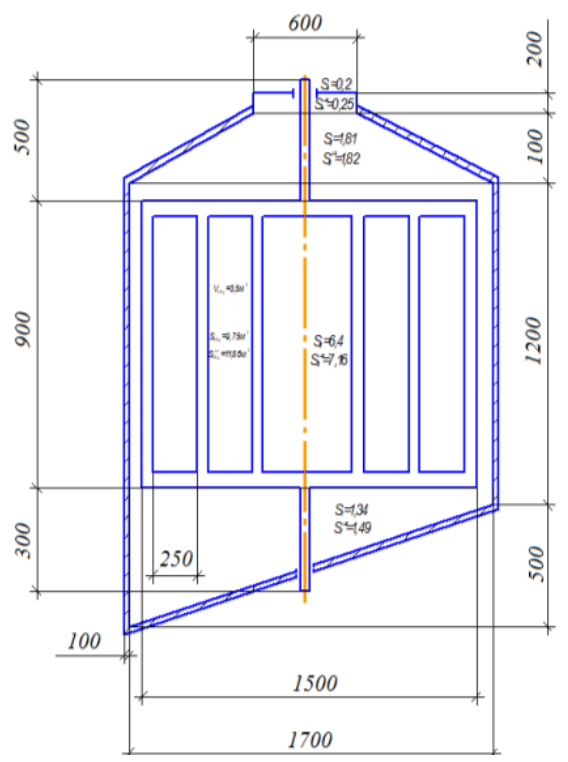

Fig. 3. Geometric parameters of a methane tank and a heat exchanger-mixer. 


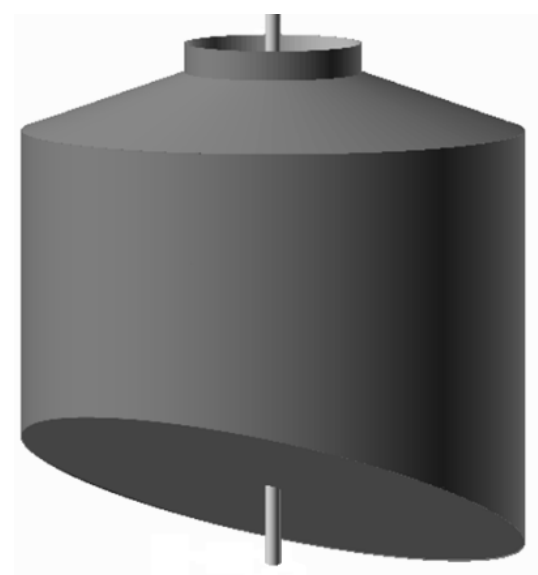

Fig. 4. Three-dimensional model of bioreactor.

An experimental sample with a $3.5 \mathrm{~m} 3$ bioreactor volume was manufactured and tested in the laboratory "Alternative Energy" of the Kabardino-Balkaria State Agrarian and showed high qualities of anaerobic fermentation of poultry waste [10].

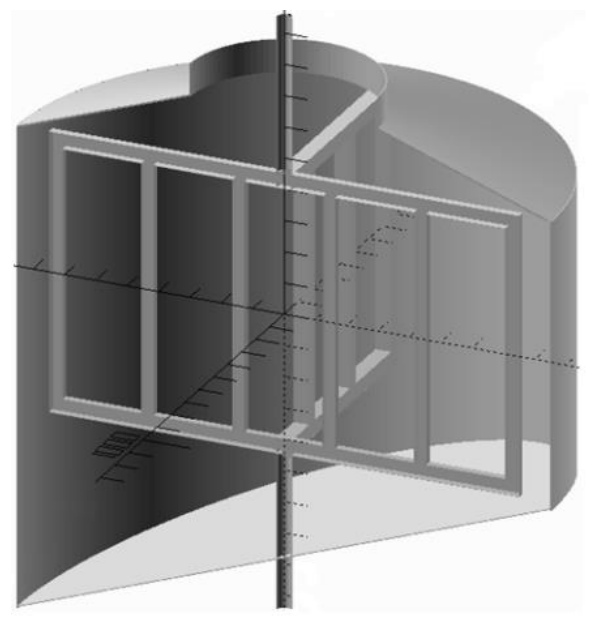

Fig. 5. The design scheme of the bioreactor.

\section{Research Questions}

Mathematical modeling is a very important stage of scientific research, since it allows us to represent the physics of the process taking place in a particular object. The most accurate and complete model helps to simplify the process of analyzing the dynamic characteristics of a physical phenomenon or process occurring in an object of research, if it is verified for the adequacy to the actual process. Expediency of mathematical modeling is caused by the high cost of conducting experiments on a real bioreactor (if it is necessary to design and manufacture it) and considerable time-consuming, due to the high inertia of thermal processes, using the example of a bioreactor, because of the large overall dimensions of the installation. 
The proposed design of the bioreactor in comparison with the prototype and other technical solutions has the following advantages:

- simplicity and small metal capacity of the structure;

- ability to perform simultaneous heating and mixing of biomass;

- $\quad$ ability to reduce energy costs for biomass heating [11];

- ability to increase the collected amount of biogas [12].

\section{Purpose of the Study}

Let's consider the thermal processes taking place in the biogas plant. The heat required for the installation in thermophilic mode, after it is put into operation, is determined by the heat loss of the installation itself. Heat exchange processes take place in the bioreactor directly in the installation volume with an increase in the litter temperature to the temperature of the thermophilic regime $\left(55-60^{\circ} \mathrm{C}\right)$, which can be described by the equations of thermodynamics.

Let's consider the process of forming a mathematical model, for a part of the bioreactor, which would make it easier to study the thermal processes taking place in this installation.

\section{Research Methods}

In order to describe the mathematical model of thermal processes, we represent the simulated part of the bioreactor in the form presented in Figure 6.

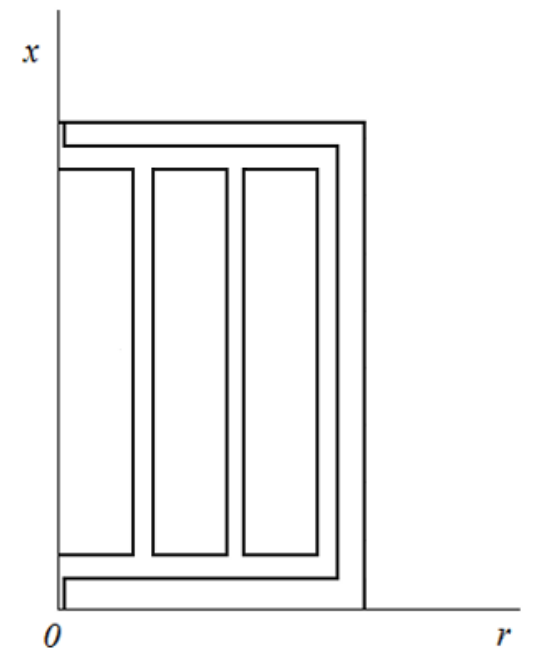

Fig. 6. Simplified scheme of the bioreactor part.

To describe a mathematical model in a cylindrical coordinate system, we introduce the basic notation: $x, r$ - spatial coordinates; $\tau$ - time; $R(\alpha), \alpha=1,2, \ldots, 8, \Delta R_{M}, \Delta R_{R}$ - basic geometric parameters of the camera along the axis $r ; X(\alpha), \alpha=1,2, \ldots, 5, \Delta X_{M}$ - basic geometric parameters of the camera along the axis $x$.

We introduce the notation introduced into the scheme shown in Figure 7. 


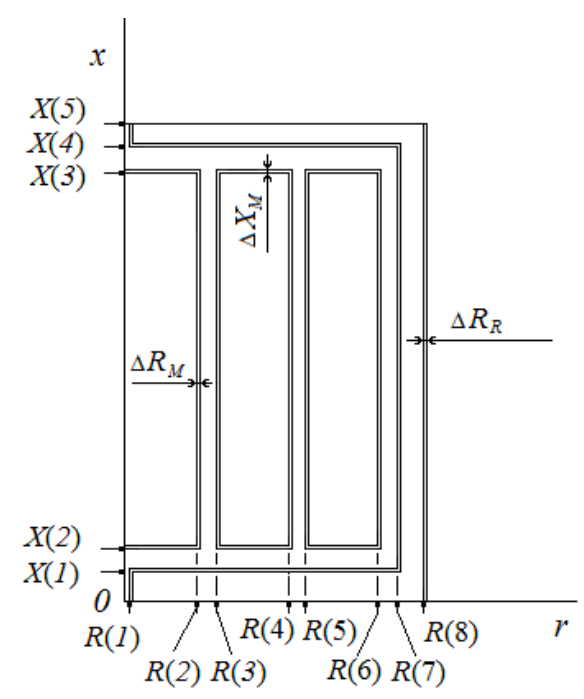

Fig. 7. Geometric parameters of the modeling object.

The system of heat conduction equations for the object under consideration can be represented in the form:

$$
\begin{aligned}
& \frac{\partial T}{\partial \tau}=a(\beta) \cdot \nabla^{2} T, T=f(x, r, \tau), \beta=1,2, \ldots, 4, \\
& \nabla^{2}=\frac{\partial^{2}}{\partial x^{2}}+\frac{1}{r} \frac{\partial}{\partial r}+\frac{\partial^{2}}{\partial r^{2}}, \tau>0 \\
& \text { If } \beta=1 \text {, then: } \\
& \text { 1) } \frac{\partial T}{\partial \tau}=a(\beta) \cdot \nabla^{2} T-\vartheta \frac{\partial T}{\partial x}, O<x<X(1)+\Delta X_{M}, O<r<R(1) \text {; } \\
& \text { 2) } \frac{\partial T}{\partial \tau}=a(\beta) \cdot \nabla^{2} T-\vartheta \frac{\partial T}{\partial r}, X(1)+\Delta X_{M} \leq x<X(2), 0<r<R(7) \text {; } \\
& \text { 3) } \frac{\partial T}{\partial \tau}=a(\beta) \cdot \nabla^{2} T-\vartheta \frac{\partial T}{\partial x}, X(2)+\Delta X_{M} \leq x \leq X(3)+\Delta X_{M} \text {, } \\
& R(2)+\Delta R_{M}<r<R(3) \\
& \text { 4) } \frac{\partial T}{\partial \tau}=a(\beta) \cdot \nabla^{2} T-\vartheta \frac{\partial T}{\partial x}, X(2)+\Delta X_{M} \leq x \leq X(3)+\Delta X_{M} \text {, } \\
& R(4)+\Delta R_{M}<r<R(5)
\end{aligned}
$$




$$
\begin{aligned}
& \text { 5) } \frac{\partial T}{\partial \tau}=a(\beta) \cdot \nabla^{2} T-\vartheta \frac{\partial T}{\partial x}, X(2)+\Delta X_{M} \leq x \leq X(3)+\Delta X_{M} \text {, } \\
& R(6)+\Delta R_{M}<r<R(7)
\end{aligned}
$$

If $\beta=2$, then:

8) $0<x<X(1), R(1)+\Delta R_{M}<r<R(8)$;

9) $X(1) \leq x \leq X(3)+\Delta X_{M}, R(7)+\Delta R_{M}<r<R(8)$;

10) $X(4)+\Delta X_{M}<x<X(5), R(1)+\Delta R_{M}<r<R(8)$;

11) $X(2)+\Delta X_{M}<x<X(3), O<r<R(2)$;

12) $X(2)+\Delta X_{M}<x<X(3), R(3)+\Delta R_{M}<r<R(4)$;

13) $X(2)+\Delta X_{M}<x<X(3), R(5)+\Delta R_{M}<r<R(6)$.

$$
\text { If } \beta=3 \text {, then: }
$$

14) $0<x \leq X(1), R(1)<r<R(1)+\Delta R_{M}$;

15) $X(1)<x<X(1)+\Delta X_{M}, R(1)<r<R(7)+\Delta R_{M}$;

16) $X(1)+\Delta X_{M}<x<X(4), R(7)<r<R(7)+\Delta R_{M}$;

17) $X(4)<x<X(4)+\Delta X_{M}, R(1)<r<R(7)+\Delta R_{M}$;

18) $X(4)+\Delta X_{M} \leq x<X(5), R(1)<r<R(1)+\Delta R_{M}$;

19) $X(2)<x<X(2)+\Delta X_{M}, O<r<R(2)+\Delta R_{M}$;

20) $X(2)+\Delta X_{M} \leq x \leq X(3), R(2)<r<R(2)+\Delta R_{M}$;

21) $X(3)<x<X(3)+\Delta X_{M}, O<r<R(2)+\Delta R_{M}$;

22) $X(2)<x<X(2)+\Delta X_{M}, R(3)<r<R(4)+\Delta R_{M}$; 
23) $X(2)+\Delta X_{M} \leq x \leq X(3), R(3)<r<R(3)+\Delta R_{M}$;

24) $X(2)+\Delta X_{M} \leq x \leq X(3), R(4)<r<R(4)+\Delta R_{M}$;

25) $X(3)<x<X(3)+\Delta X_{M}, R(3)<r<R(4)+\Delta R_{M}$;

26) $X(2)<x<X(2)+\Delta X_{M}, R(5)<r<R(6)+\Delta R_{M}$;

27) $X(2)+\Delta X_{M} \leq x \leq X(3), R(5)<r<R(5)+\Delta R_{M}$;

28) $X(2)+\Delta X_{M} \leq x \leq X(3), R(6)<r<R(6)+\Delta R_{M}$;

29) $X(3)<x<X(3)+\Delta X_{M}, R(5)<r<R(6)+\Delta R_{M}$.

$$
\text { If } \beta=4 \text {, then }
$$

$$
\text { 30) } 0<x<X(5), R(8)<r<R(8)+\Delta R_{R} \text {. }
$$

To solve the resulting system of partial differential equations, we describe the boundary and boundary conditions.

We write the boundary conditions in the form of the following system of equations.

Due to the fact that the outer part of the chamber is thermally insulated, we obtain:

$$
\frac{\partial T(x, r, \tau)}{\partial r}=0,0 \leq x \leq X(5), r=R(8)+\Delta R_{R}, \tau \geq 0 .
$$

In accordance with the symmetry condition, in the center of the chamber, the following condition is satisfied:

$$
\frac{\partial T(x, r, \tau)}{\partial r}=0,0 \leq x \leq X(5), \tau \geq 0 .
$$

Above and below, in accordance with the characteristics of the reactor, the following conditions are accepted:

$$
\begin{gathered}
\lambda(l) \frac{\partial T(x, r, \tau)}{\partial x}=q 1(\tau), x=X(5), 0 \leq r \leq R(1), \tau \geq 0 \\
-\lambda(l) \frac{\partial T(x, r, \tau)}{\partial x}=q 2(\tau), x=X(5), R(l)<r \leq R(8)+\Delta R_{R}, \tau \geq 0 ; \\
-\lambda(l) \frac{\partial T(x, r, \tau)}{\partial x}=q 3(\tau), x=0,0 \leq r \leq R(8)+\Delta R_{R}, \tau \geq 0 .
\end{gathered}
$$

Next, we write the conditions on the media interfaces $(\tau \geq 0)$ : 


$$
\begin{aligned}
& S 5: \lambda(2) \frac{\partial T(x, r, \tau)}{\partial r}=\lambda(3) \frac{\partial T(x, r, \tau)}{\partial r}, O<x \leq X(1)+\Delta X_{M}, r=R(1) \text {; } \\
& S 6: \lambda(3) \frac{\partial T(x, r, \tau)}{\partial r}=\lambda(l) \frac{\partial T(x, r, \tau)}{\partial r}, 0<x \leq X(1), r=R(l)+\Delta R_{M} ; \\
& S 7: \lambda(3) \frac{\partial T(x, r, \tau)}{\partial x}=\lambda(l) \frac{\partial T(x, r, \tau)}{\partial x}, x=X(l), \\
& R(1)+\Delta R_{R} \leq r \leq R(7)+\Delta R_{M} ; \\
& S 8: \lambda(2) \frac{\partial T(x, r, \tau)}{\partial x}=\lambda(3) \frac{\partial T(x, r, \tau)}{\partial x}, x=X(1)+\Delta X_{M}, R(1) \leq r \leq R(7) ; \\
& S 9: \lambda(3) \frac{\partial T(x, r, \tau)}{\partial r}=\lambda(1) \frac{\partial T(x, r, \tau)}{\partial r}, X(1) \leq x \leq X(4)+\Delta X_{M}, r=R(7)+\Delta R_{M} \text {; } \\
& \text { S10: } \lambda(3) \frac{\partial T(x, r, \tau)}{\partial r}=\lambda(2) \frac{\partial T(x, r, \tau)}{\partial r}, X(1)+\Delta X_{M} \leq x \leq X(4), r=R(7) \text {; } \\
& \text { S11: } \lambda(3) \frac{\partial T(x, r, \tau)}{\partial x}=\lambda(1) \frac{\partial T(x, r, \tau)}{\partial x}, x=X(4)+\Delta X_{M}, \\
& R(1)+\Delta R_{R} \leq r \leq R(7)+\Delta R_{M} ; \\
& S 12: \lambda(2) \frac{\partial T(x, r, \tau)}{\partial x}=\lambda(3) \frac{\partial T(x, r, \tau)}{\partial x}, x=X(4), R(1) \leq r \leq R(7) \text {; } \\
& S 13: \lambda(3) \frac{\partial T(x, r, \tau)}{\partial r}=\lambda(1) \frac{\partial T(x, r, \tau)}{\partial r}, X(4)+\Delta X_{M} \leq x \leq X(5), \\
& r=R(1)+\Delta R_{M} \\
& S 14: \lambda(2) \frac{\partial T(x, r, \tau)}{\partial r}=\lambda(3) \frac{\partial T(x, r, \tau)}{\partial r}, X(4) \leq x \leq X(5), r=R(1) ; \\
& S 15: \lambda(2) \frac{\partial T(x, r, \tau)}{\partial x}=\lambda(3) \frac{\partial T(x, r, \tau)}{\partial x}, x=X(2), 0 \leq r \leq R(2)+\Delta R_{M} ; \\
& \text { S16: } \lambda(3) \frac{\partial T(x, r, \tau)}{\partial x}=\lambda(1) \frac{\partial T(x, r, \tau)}{\partial x}, x=X(2)+\Delta X_{M}, 0 \leq r \leq R(2) \text {; } \\
& S 17: \lambda(1) \frac{\partial T(x, r, \tau)}{\partial r}=\lambda(3) \frac{\partial T(x, r, \tau)}{\partial r}, X(2)+\Delta X_{M} \leq x \leq X(3), r=R(2) \text {; }
\end{aligned}
$$




$$
\begin{aligned}
& S 18: \lambda(2) \frac{\partial T(x, r, \tau)}{\partial r}=\lambda(3) \frac{\partial T(x, r, \tau)}{\partial r}, X(2) \leq x \leq X(3)+\Delta X_{M} \text {, } \\
& r=R(2)+\Delta R_{M} \\
& S 19: \lambda(2) \frac{\partial T(x, r, \tau)}{\partial x}=\lambda(3) \frac{\partial T(x, r, \tau)}{\partial x}, x=X(3), O \leq r \leq R(2)+\Delta R_{M} \text {; } \\
& S 20: \lambda(3) \frac{\partial T(x, r, \tau)}{\partial x}=\lambda(l) \frac{\partial T(x, r, \tau)}{\partial x}, x=X(3)+\Delta X_{M}, 0 \leq r \leq R(2) \text {; } \\
& S 21: \lambda(2) \frac{\partial T(x, r, \tau)}{\partial x}=\lambda(3) \frac{\partial T(x, r, \tau)}{\partial x}, x=X(2), R(3) \leq r \leq R(4)+\Delta R_{M} ; \\
& S 22: \lambda(3) \frac{\partial T(x, r, \tau)}{\partial x}=\lambda(1) \frac{\partial T(x, r, \tau)}{\partial x}, x=X(2)+\Delta X_{M}, \\
& R(3)+\Delta R_{M} \leq r \leq R(4) \\
& S 23: \lambda(2) \frac{\partial T(x, r, \tau)}{\partial r}=\lambda(3) \frac{\partial T(x, r, \tau)}{\partial r}, X(2) \leq x \leq X(3)+\Delta X_{M}, r=R(3) \text {; } \\
& S 24: \lambda(1) \frac{\partial T(x, r, \tau)}{\partial r}=\lambda(3) \frac{\partial T(x, r, \tau)}{\partial r}, X(2)+\Delta X_{M} \leq x \leq X(3), \\
& r=R(3)+\Delta R_{M} \\
& S 25: \lambda(1) \frac{\partial T(x, r, \tau)}{\partial r}=\lambda(3) \frac{\partial T(x, r, \tau)}{\partial r}, X(2)+\Delta X_{M} \leq x \leq X(3), r=R(4) ; \\
& S 26: \lambda(2) \frac{\partial T(x, r, \tau)}{\partial r}=\lambda(3) \frac{\partial T(x, r, \tau)}{\partial r}, X(2) \leq x \leq X(3)+\Delta X_{M}, r=R(4)+\Delta R_{M} \text {; } \\
& S 27: \lambda(2) \frac{\partial T(x, r, \tau)}{\partial x}=\lambda(3) \frac{\partial T(x, r, \tau)}{\partial x}, x=X(3), R(3) \leq r \leq R(4)+\Delta R_{M} ; \\
& S 28: \lambda(3) \frac{\partial T(x, r, \tau)}{\partial x}=\lambda(1) \frac{\partial T(x, r, \tau)}{\partial x}, x=X(3)+\Delta X_{M}, R(3)+\Delta R_{M} \leq r \leq R(4) ; \\
& S 29: \lambda(2) \frac{\partial \mathrm{T}(\mathrm{x}, \mathrm{r}, \tau)}{\partial x}=\lambda(3) \frac{\partial \mathrm{T}(\mathrm{x}, \mathrm{r}, \tau)}{\partial x}, x=X(2), R(5) \leq r \leq R(6)+\Delta R_{M} ; \\
& S 30: \lambda(3) \frac{\partial \mathrm{T}(\mathrm{x}, \mathrm{r}, \tau)}{\partial x}=\lambda(1) \frac{\partial \mathrm{T}(\mathrm{x}, \mathrm{r}, \tau)}{\partial x}, x=X(2)+\Delta X_{M}, R(5)+\Delta R_{M} \leq r \leq R(6) ; \\
& S 31: \lambda(2) \frac{\partial T(x, r, \tau)}{\partial r}=\lambda(3) \frac{\partial T(x, r, \tau)}{\partial r}, X(2) \leq x \leq X(3)+\Delta X_{M}, r=R(5) ;
\end{aligned}
$$




$$
\begin{gathered}
S 32: \lambda(1) \frac{\partial T(x, r, \tau)}{\partial r}=\lambda(3) \frac{\partial T(x, r, \tau)}{\partial r}, X(2)+\Delta X_{M} \leq x \leq X(3), r=R(5)+\Delta R_{M} ; \\
S 33: \lambda(1) \frac{\partial T(x, r, \tau)}{\partial r}=\lambda(3) \frac{\partial T(x, r, \tau)}{\partial r}, X(2)+\Delta X_{M} \leq x \leq X(3), r=R(6) ; \\
S 34: \lambda(2) \frac{\partial T(x, r, \tau)}{\partial r}=\lambda(3) \frac{\partial T(x, r, \tau)}{\partial r}, X(2) \leq x \leq X(3)+\Delta X_{M}, r=R(6)+\Delta R_{M} ; \\
S 35: \lambda(2) \frac{\partial T(x, r, \tau)}{\partial x}=\lambda(3) \frac{\partial T(x, r, \tau)}{\partial x}, x=X(3), R(5) \leq r \leq R(6)+\Delta R_{M} ; \\
S 36: \lambda(3) \frac{\partial T(x, r, \tau)}{\partial x}=\lambda(1) \frac{\partial T(x, r, \tau)}{\partial x}, x=X(3)+\Delta X_{M}, R(5)+\Delta R_{M} \leq r \leq R(6) ; \\
S 37: \lambda(1) \frac{\partial \mathrm{T}(\mathrm{x}, \mathrm{r}, \tau)}{\partial \mathrm{r}}=\lambda(4) \frac{\partial \mathrm{T}(\mathrm{x}, \mathrm{r}, \tau)}{\partial \mathrm{r}}, 0 \leq x \leq X(4), r=R(8),
\end{gathered}
$$

where: $\Delta R_{R}$ - bioreactor wall thickness; $\Delta X_{M}$ - the wall thickness of the heat exchangermixer blades; $q 1(\tau)$ - heat flow in the heat exchanger-mixer; $q 2(\tau), q 3(\tau)$ - heat losses; $\vartheta$ coolant flow rate.

\section{Findings}

The simulation was carried out on the assumption that at the initial time the temperature was uniformly distributed throughout the chamber, i.e. $T(x, r, \tau=0)=$ const $=0$.

Coefficients of thermal diffusivity $a(\beta)$ materials and substances are determined from the following relation:

$$
a(\beta)=\frac{\lambda(\beta)}{c(\beta) \cdot \rho(\beta)},\left[\begin{array}{c}
M^{2} \\
c е \kappa
\end{array}\right], \beta=1,2, \ldots, 4,
$$

where $\rho\left[\frac{\kappa 2}{M^{3}}\right]$ - matter density, $\lambda\left[\frac{B m}{M \cdot \tau p a d}\right]$ - coefficient of thermal conductivity of matter,

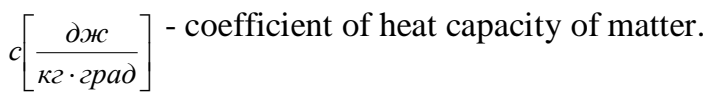

Density, heat capacity, and thermal conductivity of substances are determined from tables [13].

Table 1. Thermal conductivity, density, and heat capacity of substances.

\begin{tabular}{|c|c|c|c|}
\hline Substance & $\begin{array}{c}\text { Thermal } \\
\text { conductivity } \\
\lambda\left[\frac{B m}{M \cdot \tau p a d}\right]\end{array}$ & $\begin{array}{c}\text { Density } \\
\rho_{\Pi}\left[\frac{\kappa 2}{M^{3}}\right]\end{array}$ & $\begin{array}{c}\text { Heat capacity } \\
c\left[\frac{\partial \varkappa c}{\kappa 2 \cdot 2 p a d}\right]\end{array}$ \\
\hline Water & 0,561 & 915 & 4,179 \\
\hline
\end{tabular}




\begin{tabular}{|c|c|c|c|}
\hline Air & 0,0075 & 1,32 & 960 \\
\hline Heat-resistant steel & 54,4 & $7760-8000$ & 486 \\
\hline
\end{tabular}

For the linearization of the model, we average the values of the coefficients given in the tables and determine the coefficients of thermal diffusivity of materials and substances from which the structural elements of the heat exchanger-mixer are made and take part in the process of heat exchange in the bioreactor:

- heat-resistant steel

$$
\alpha(3)=\alpha(4)=\frac{54,4}{7800 \cdot 486}=1,43505 \cdot 10^{-5},\left[\begin{array}{c}
M^{2} \\
\text { сек }
\end{array}\right]
$$

- air

$$
a(1)=\frac{0,0075}{960 \cdot 1,32}=0,0000059,\left[\frac{\mathcal{M}^{2}}{c e \kappa}\right]
$$

- water

$$
a(2)=\frac{0,561}{4,195 \cdot 915}=0,0004291,\left[\begin{array}{c}
M^{2} \\
c e \kappa
\end{array}\right] .
$$

All the obtained data is provided in the following table:

Table 2. Coefficients of thermal conductivity and thermal diffusivity of substances.

\begin{tabular}{|c|c|c|}
\hline Substance & Thermal conductivity & Thermal diffusivity \\
\hline $\begin{array}{c}\text { Chromium } \\
\text { molybdenum } \\
\text { steel }\end{array}$ & 54,4 & $1,43505 \cdot 10^{-5}$ \\
\hline Air & 0,0075 & 0,0000059 \\
\hline Water & 0,561 & 0,0004291 \\
\hline
\end{tabular}

\section{Conclusion}

An analysis of the above equations shows that the main influence on the heat transfer process is due to the thermal conductivity, thermal diffusivity, density, and structural and technological parameters of the heat exchanger-mixer. The latter characterizes the intensity of the forced motion of fermentable manure, while changing the value of thermal conductivity.

Thus, the mixing time and, consequently, the degree of temperature uniformity of fermentable manure depends on the multiplicity of biomass circulation in the plant. The theoretical temperature homogeneity of the stirred mass is achieved with the circulation number of the heat exchanger-mixer tending to infinity, and the permissible degree of temperature homogeneity corresponds to values not lower 0,96 [14].

It is established that the theoretical temperature homogeneity of the stirred medium is achieved by combining the heat exchanger and the mixing device into one unit, which allows to heat and maintain a given temperature regime more evenly throughout the volume of the 
onsite sewage facility. As a result of theoretical studies of the heat transfer and heat transfer processes taking place in the biogas plant, a mathematical model has been obtained that makes it possible to determine the distribution of the temperature of the biomass throughout the entire volume of the onsite sewage facility.

The design and technological scheme and parameters of the biogas plant differ from the known ones in that the agitator (stirrer) and the heating element are combined into one unit, that is, the stirring device is simultaneously a heating element. This combination allows heating and maintaining a given temperature regime more evenly due to the rotation of the heat exchanger and the transfer of heat to the biomass (substrate) throughout the entire volume of the onsite sewage facility.

\section{References}

1. A. G. Fiapshev, O. Kh. Kilchukova, A. I. Yurov, VIESH Insitute Herald, 4, 17 (2014)

2. T. B. Temukuev, A. G. Fiapshev, Economic and technical mechanisms to promote energy conservation (Polygraphservice and T, Nalchik, 2009)

3. A. I, Yurov, A. G. Fiapshev, O. Kh. Kilchukova, Herald of the Agrarian and Industrial Complex of Stavropol, 3, 15 (2014)

4. O. Kh. Kilchukova, Rural Mechanic, 7 (2017)

5. A. G. Fiapshev, O. Kh. Kilchukova, M. M. Khamokov, Materials of the International Scientific and Practical Conference on Energy Supply and Energy Savings in Agriculture (SSA ARIAE, Moscow, 2014)

6. A. G. Fiapshev, O. Kh. Kilchukova, M. M. Hamokov, Rural Mechanic, 2 (2017)

7. A. G. Fiapshev, O. Kh. Kilchukova, A. K. Apazhev, L. M. Khazhmetov, Yu. A. Shekikhachev, M. M. Khamokov, Bioreactor. Patent No. 152918. 03/03/2015. Byul., 17 (2015)

8. A. G. Fiapshev, O. Kh. Kilchukova, M. M. Khamokov, Energy Safety and Energy Economy, 2 (2017)

9. A. G. Fiapshev, O. Kh. Kilchukova, M. M. Khamokov, B. B. Temukuev, Herald of the Agroindustrial Complex of Stavropol Territory, 3, 19 (2015)

10. O. Kh. Kilchukova, A. G. Fiapshev, M. M. Khamokov, Materials of the All-Russian Scientific and Practical Conference with International Participation on Actual Problems in Energy and Means of Mechanization of Agroindustrial Complex (Blagoveshensk, 2014)

11. A. G. Fiapshev, O. Kh. Kilchukova, Izvestiya of the Nizhnevolzhsk Agro-University Complex: Science and Higher Vocational Education, 3, 39 (2015)

12. A. G. Fiapshev, O. Kh. Kilchukova, TB Temukuev, MM Khamokov, Izvestiya of the Gorskiy State Agrarian University, 51, 4 (2014)

13. I. S. Grigorieva, E. Z. Meilikhova, Physical quantities: reference book (Energoatomizdat, Moscow, 1991)

14. E. A. Vasiltsov, Apparatus for mixing liquid media (Mashinostroyeniye, Moscow, 1979)

15. A. V. Bogoviz, S. V. Lobova, Y. A. Bugai, Advances in Intelligent Systems and Computing, 622, 30-36 (2018)

16. A. V. Bogoviz, S. V. Lobova, Y. V. Ragulina, A. N. Alekseev, International Journal of Energy Economics and Policy, 8(3), 67-75 (2018) 